\title{
LA FORMACIÓN INICIAL Y PERMANENTE DEL PROFESORADO DE EDUCACIÓN FÍSICA A TRAVÉS DEL PRÁCTICUM.
}

\author{
Herminia Ma García Ruso \\ Universidad de Santiago de Compostela
}

RESUMEN: En este artículo se muestran los aspectos más importantes del desarrollo de un proyecto de innovación educativa realizado durante las prácticas de enseñanza. En el proyecto intervinieron cuatro alumnos, futuros maestros especialistas de Educación Física, cuatro tutoras y dos supervisoras. Trataremos de exponer, con cierto matiz descriptivo, las fases seguidas en la realización de un proyecto de investigación-acción, los objetivos formulados, la metodología empleada, la elaboración de una unidad didáctica de expresión corporal y el análisis e interpretación de la información. Finalmente, explicaremos las conclusiones que giran alrededor de tres ejes: la relación de la Universidad y los Centros Educativos, la relación del supervisor y el tutor, y la valoración del propio seminario de investigación-acción como herramienta para la investigación y la formación inicial y permanente del profesorado.

ABSTRACT: This article shows the most important aspects of the development of a collaborative research-action project carried out while future Physical Education teachers were actually giving classes at schools. Members of the group working on the project were four students specializing in Physical Education, four tutors and two supervisors. We have tried, with some descriptive nuances, to explain the phases followed in carrying out this researchaction project, the aims formulated, the methodology used, the working-out of a didactic unit of body expression, and the analysis and interpretation of the information. Finally, we explain the conclusions that revolve around from three central ideas -the relationship University-Educational centres, the relationship supervisor-tutor and the appraisal of the research-action seminar itselfas tools for research and the initial and permanent education of teachers.

PALABRAS CLAVE: Educación Física, formación de profesores de educación física, prácticum, investigación-acción.

KEYWORDS: Physical Education, teacher education in physical education, prácticum and research-action. 


\section{INTRODUCCIÓN}

Desde comienzos de la década de los noventa, la finalidad de nuestra investigación se ha centrado siempre en torno a la formación inicial del profesorado de Educación Física. En este período, hemos dedicado una creciente atención a este tema procurando siempre buscar referencias que nos dieran pie para incorporar nuevas dimensiones a nuestra línea de investigación. En el cruce de intereses y motivaciones surge la investigación aquí desarrollada que pone en relación la formación inicial, la formación permanente y el prácticum.

\section{MARCO TEÓRICO}

El objetivo de este apartado es exponer, someramente, los principales pilares teóricos que sustentan la presente investigación, a saber:

- La formación inicial y permanente del Profesorado de Educación Física.

- El prácticum.

- La investigación-acción.

\subsection{La formación inicial y permanente del Profesorado de Educación Física}

La preparación de profesores reflexivos ha sido una meta de los programas de formación durante mucho tiempo. Son varios los formadores de profesores que, aun cuando saben que fomentar la reflexión no es una tarea fácil, tienen como objetivo de sus programas preparar a los docentes para que sean capaces de pensar y reflexionar sobre sus acciones. Los esfuerzos por apoyar y permitir a los profesores que reflexionen sobre el proceso de enseñanza-aprendizaje y otros problemas educativos debe empezar en la formación inicial y continuar a lo largo de su preparación profesional (véanse, entre otros, Almond, 1997; Blández, 1996; Fraile, 1997; García Ruso, 1993, 1997, 2002; Graham, 1991; Hardy, 1999; Martínez y Vaca, 1999; Rovegno, 1992; Tinning, 1994; Toja, 2001; Tsangaridou \& O’Sullivan, 1994; Viciana, 1996).

Esta amplia conceptualización presente en nuestra investigación está asentada dentro de un marco explícito, y con la asunción de un determinado paradigma de formación del profesorado. El modelo de formación de profesores que les concede un protagonismo activo en su formación y que a su vez fomenta la crítica respecto al currículum, nos orienta hacia la asunción de un paradigma interpretativo. Paradigma que asumimos como un elemento integrador de nuestro marco teórico de referencia, con la pretensión primordial de que nos facilite las relaciones entre la formación inicial y la formación permanente del profesorado, mediante la interconexión de la relación dialéctica de la teoría y la práctica y siempre bajo la dimensión del pensamiento reflexivo y crítico del profesor. Si esto acontece, los profesores progresarán en la transformación de su práctica, incorporando teorías y estrategias que provengan de su propia experiencia docente individual y colaborativa. De ahí que nuestra visión de la formación del profesorado no se establezca dentro de un ámbito cerrado y concluso, sino, muy al contrario, en un ámbito donde las coordenadas primordiales sean: la formación inicial y permanente, la renovación pedagógica, la calidad de la enseñanza y la innovación e investigación educativa. 


\subsection{El prácticum}

Definimos el prácticum como experiencias orientadas, que pretenden que el alumno, futuro profesor, utilice, de manera crítica y reflexiva, los conocimientos teórico-prácticos apropiados a las circunstancias concretas que se le pueden presentar en el proceso de enseñanza-aprendizaje y en el contexto escolar. Con los términos experiencias orientadas queremos hacer referencia a la planificación en el sentido que le da Carreiro $(1994$, p.1) cuando señala que la etapa de prácticas incluye todas las actividades de formación y preparación planificadas por las instituciones o por los propios profesores para propiciar su desarrollo profesional y la mejora de su capacidad como docente.

Las prácticas de enseñanza o prácticum no son tan sólo una ocasión para que los futuros profesores aprendan a enseñar, sino que, además, ofrecen una gran oportunidad para la formación continua de los profesores tutores y supervisores. En este sentido, Díaz y Martínez (1994, p.100) hacen referencia a los alumnos, profesores tutores y supervisores como tres vértices que componen las prácticas, afirmando que si estos funcionan como un equipo, las prácticas adquieren gran calidad, pero cuando cada uno busca diferentes cosas en ellas, no pasan de ser un trámite, a veces con resultados perjudiciales. Las prácticas para que ayuden al profesional a ser cada día más reflexivo deben estar coordinadas con seminarios, clases, debates; es decir, escenarios donde al alumno en prácticas pueda aprender como utilizar los medios a su alcance y, además, llegar a una observación más reflexiva y participante (entre otros autores, cabría citar, Fraile, 1997; García Ruso, 1997, 2002; Martínez, 2001; Ortiz y Romero, 2000; Romero, 1995; Toja, 2001).

Durante la realización del prácticum se hacen más evidentes las dificultades de la relación teoría-práctica. Si todo aquello que adquirimos teóricamente se pudiera transferir con éxito a la práctica, bastaría con tener buena y abundante información. Sin embargo, sabemos que esto realmente no es así. A la sincronización entre la intención, saber teórico, y la realización, práctica en el aula, subyace una relación compleja. El interés por la coordinación entre la formación teórica y práctica del profesorado es una persistente idea que se viene manteniendo viva desde hace mucho tiempo en la historia de la formación inicial de los docentes.

En la formación inicial del profesor el tema de la relación de la teoría y la práctica es de enorme interés (Marcelo 1995; Marcelo y Estebaranz, 1998; Montero, 1990; Paiva, 1998; Zabalza, 1993). Por ello, en el desarrollo del currículum formativo es necesario optar por un modelo que permita al alumno, futuro profesor, tomar parte en el proceso de enseñanza-aprendizaje de manera activa, crítica y reflexiva. En nuestra opinión, la interacción teoría-práctica no debe tener lugar tan sólo en el momento secuencial de las prácticas, sino que debe estar presente en todo el proceso curricular, a través de una metodología crítica y reflexiva. Los docentes reflexivos no sólo se caracterizan por su habilidad de pensar críticamente, sino también por la asociación del pensamiento con la acción. De ahí la importancia que tiene que el futuro profesor tenga oportunidades para desarrollar y refinar sus habilidades reflexivas. O’Sullivan \& Tsangaridou (1997, p.9)) arguyen que el proceso de reflexión informa a los maestros sobre lo que necesita ser cambiado, y cuándo y cómo estos cambios necesitan ser hechos para reforzar y facilitar el proceso de aprendizaje.

Las prácticas de enseñanza deben entenderse como una situación privilegiada para la formación no sólo del futuro profesor, sino también del profesor tutor y supervisor. Esta idea de prácticum lleva consigo implícitamente un trabajo de colabora- 
ción entre los profesionales de la institución escolar y los profesionales de la universidad. Esta relación de colaboración no se debería establecer únicamente para realizar las prácticas, sino que debería estar integrada en la cultura formativa de ambas instituciones a través de proyectos de innovación educativa. En tal sentido y teniendo siempre presente la idea de conseguir ser un profesional reflexivo, consideramos que los seminarios de investigación-acción, donde colaboren profesionales de otros niveles educativos y alumnos en prácticas, constituyen un medio primordial para vincular la teoría y la práctica y estimular la reflexión en unión estrecha con la práctica docente y la investigación.

\subsection{La investigación-acción}

Revisando la historia de la investigación-acción (véanse Zeichner \& Noffke, 2001, Mckernan, (1999) se puede asociar su renacer a investigadores como Stenhouse $(1975,1987)$ y Elliott $(1990,1993)$. De sus obras se deduce que la investigaciónacción no es sólo investigación, es también un proceso de formación de profesores. En la década de los años ochenta comienzan a surgir nuevos enfoques ligados a la teoría crítica. Es de obligado rigor citar aquí, entre otros, a Carr \& Kemmis (1988) y Kemmis \& Mctaggart (1988). Algunas de las razones para este resurgimiento fueron:

- Un nuevo interés en ayudar a los profesores a mejorar su práctica docente.

- La colaboración de las universidades y las escuelas en proyectos comunes de investigación.

- La creencia de que la investigación educativa tradicional era incapaz de resolver los problemas educativos.

La investigación - acción puede ser considerada como una modalidad metodológica con un enorme potencial de enriquecimiento para el campo de la investigación educativa. Es una forma de entender la práctica docente que proporciona a los profesores un medio idóneo para desarrollar dialécticamente la teoría y la práctica. Las actividades de investigación - acción ayudan a los implicados no sólo a resolver los problemas que la práctica educativa conlleva, sino que, además, contribuyen en palabras de Carr \& Kemmis (1988, p. 190) a que los docentes superen los autoentendimientos distorsionados mediante el análisis de cómo sus propias prácticas y entendimientos están configurados por condiciones ideológicas más amplias.

Para considerar la investigación - acción como tal, debe reunir ciertas condiciones, a saber:

- Debe ocuparse de investigar sistemáticamente una práctica social o educativa, focalizando la atención en los problemas del mundo real de los profesores.

- Debe ser participativa y colaborativa, es decir, una actividad de grupo, no individualista. Los profesores e investigadores trabajan juntos, planifican, ejecutan y analizan.

- Debe utilizar la espiral de la autorreflexión, donde la investigación - acción es un continuo ciclo con el énfasis puesto en una mejora de la comprensión y de la práctica.

Considera Tinning (1992. p. 11) que la investigación - acción tiene un gran potencial para la mejora de la vida de los profesores. Pero advierte que sólo la que aspire 
a ser emancipatoria garantiza el uso del término, y la investigación colaborativa que utiliza el ciclo de la investigación - acción focalizando sólo su atención en los aspectos técnicos de la mejora de la práctica sería más conveniente describirla como otra cosa que como investigación - acción.

\section{OBJETIVOS DEL PROYECTO DE INVESTIGACIÓN-ACCIÓN}

Con los objetivos de nuestro proyecto trataremos de:

- Lograr que un pequeño grupo de profesionales de la Educación física inicie un trabajo de formación permanente, a través de un proyecto de investigaciónacción, con incidencia en la calidad de la enseñanza y del prácticum.

- Contribuir de forma práctica al desarrollo profesional de los participantes en el citado proyecto, gracias a un modelo de investigación basado en la observación, análisis y reflexión de lo que acontece en el aula, así como la posterior puesta en práctica de las soluciones oportunas.

- Revisar y reflexionar sobre las estrategias educativas que los profesores utilizan en su función docente.

- Elaborar estrategias y modelos creativos que incluyan innovaciones curriculares, metodológicas, tecnológicas y didácticas aplicables al campo de la Educación Física en la enseñanza primaria, así como su posterior puesta en práctica analizando los efectos producidos.

- Buscar nuevas formas de actuación en el aula contrastándolas con el resto de los componentes del grupo, con el objeto de mejorar la calidad de la enseñanza y el desarrollo profesional de los docentes.

- Fomentar la innovación educativa a través de la actividad investigadora de los profesores partiendo de su propia práctica docente.

- Colaborar con nuestra experiencia investigadora al desarrollo curricular de la Educación Física.

- En síntesis, con estos objetivos trataremos que un pequeño grupo de profesionales de la Educación Física inicie un trabajo de formación inicial y permanente, a través de un proyecto de investigación-acción, con la pretensión de mejorar la calidad de la enseñanza y del prácticum.

\section{DISEÑO Y METODOLOGÍA}

La metodología empleada es cualitativa, es decir, aquella que siguen los que piensan que la realidad educativa se encuentra en un continuo cambio, dando gran importancia al contexto en el que tiene lugar la investigación y al significado que los agentes implicados dan al propio proceso. En tal sentido, utilizaremos la investigación-acción crítica que propugna la relación entre la educación y los dos contextos, o sea, el triángulo: educación-aula-sociedad. Desde este punto de vista la práctica no sólo le compete al profesor, sino a todo el grupo que, de forma colaborativa, elabora y adquiere una comprensión crítica y reflexiva de las prácticas educativas. Ello conlleva la asunción de responsabilidades por parte de todos los miembros del grupo, quedando así relegado, e incluso cuestionado, el papel de expertos en la investigación, postulando así la existencia de comunidades autoreflexivas de teóricos y prácticos, comprometidos en su labor docente. 
El proceso seguido para llevar a cabo nuestro proyecto ha sido el siguiente:

Definir el equipo de trabajo: estaba formado por dos profesoras universitarias, cuatro maestras de educación primaria y cuatro alumnos, dos chicas y dos chicos, de magisterio que se encontraban realizando el prácticum en la especialidad de Educación Física con dichas maestras.

Identificación del problema: además de las motivaciones personales de cada uno de los miembros del equipo, el primer punto de referencia para delimitar el problema objeto de la investigación-acción venía en parte dado por el Plan de Prácticas y más concretamente por los objetivos de las mismas, que pueden sintetizarse en los siguientes términos:

- El conocimiento de la escuela como medio educador intencional, relacionándola con el medio socioeconómico, cultural e histórico, con la finalidad de comprender las funciones que realiza el sistema educativo en la sociedad.

- Desarrollar en el alumno la capacidad de análisis del trabajo realizado en la escuela, utilizando técnicas adecuadas.

- Las prácticas servirán para que el alumno descubra la complejidad de la tarea de la enseñanza, de tal manera que tenga recursos para transformarla u optimizarla.

- Las prácticas permitirán al alumno identificar los distintos aspectos implicados en el proceso de enseñanza-aprendizaje, desarrollando el sentido crítico y la reflexión.

- Capacitar al alumno para paulatinamente responsabilizarse de tareas colaborando con el maestro y con los equipos de profesores, basando su actuación en el respecto, la comunicación y la colaboración en los objetivos y problemas de la escuela.

- Capacitar a los alumnos para tomar decisiones con la madurez personal en el ámbito de la clase y de las actividades extraescolares.

- El alumno deberá saber analizar su propia actuación, valorando y contrastando los modelos observados con otros modelos y su propia práctica.

De acuerdo con estos objetivos y sin perder de vista los enunciados en nuestro proyecto, delimitamos junto con las profesoras tutoras y los alumnos en prácticas el tema central del proyecto, que tenía la pretensión de ser innovador en el ámbito de la formación inicial y permanente. Después de debatir, reflexionando colaborativamente todos los participantes, sobre cuales eran los propósitos del proyecto, hicimos un diagnóstico de la situación docente actual, y el grupo como tal sintió la necesidad de profundizar en el proceso de enseñanza-aprendizaje de la Educación Física. Para ello, centramos fundamentalmente nuestra atención en la construcción de los materiales curriculares y la puesta en práctica de los mismos, concretamente elaboramos una unidad didáctica, utilizando en todo el proceso la espiral de la investigación-acción.

El hecho de que tanto las profesoras supervisoras, como las maestras tutoras y alumnos en prácticas, pusieran de manifiesto las limitaciones que arrastraban en su propia formación en los contenidos curriculares relacionados con la expresión cor- 
poral, fue, sin duda, el acicate para seleccionar como tema de la unidad didáctica el de la expresión corporal.

Revisión crítica de la literatura: sobre la propia metodología que se iba a emplear y sobre el tema objeto de estudio, que en este caso era la expresión corporal. Dedicamos al inicio de la puesta en práctica del proyecto algún tiempo a la lectura de textos que considerábamos importantes para iluminar el proceso que deberíamos seguir. Consideramos al igual que Maxwell (1998) que la función de la teoría es la de informar para ayudar a formar juicios sobre los propósitos, desarrollar y seleccionar las cuestiones relevantes y realistas de la investigación, elegir los métodos coherentes con el objeto de estudio e identificar las diferentes amenazas de la validez de las conclusiones.

Planificación, acción y reflexión: A medida que avanzábamos en la planificación, intentábamos dar respuestas de manera específica y concreta a los interrogantes: ¿Qué debe hacerse a cerca de qué, por parte de quién, dónde, cuándo y cómo? Además, éramos conscientes que para que el plan se desarrollara satisfactoriamente, era necesario que las respuestas fueran compartidas por todos los miembros que colaborábamos en el equipo de investigación-acción.

\subsection{La recogida de información}

La recogida de información se realizó desde el comienzo del proyecto y continuó a lo largo de todo el proceso hasta finalizar el período de prácticas. Inicialmente, realizamos entrevistas abiertas tanto al profesor supervisor como a los profesores tutores y alumnos en prácticas. Estas entrevistas tenían como propósitos fundamentales indagar sobre el significado que cada uno de ellos daba al prácticum, conocer cuáles eran sus expectativas, el contexto particular en el que trabajaban o estudiaban y la influencia de este contexto en su labor profesional o preprofesional. La información obtenida nos servió para reflexionar sobre ella y reconducir nuestro propio proyecto. Al finalizar el prácticum mantuvimos otra entrevista con todos los participantes. Las preguntas formuladas a todos ellos giraban, fundamentalmente, entorno a dos cuestiones. En la primera, pretendíamos recabar información acerca de las relaciones entre la Universidad y los Centros Educativos y, por ende, la relación entre el tutor y el supervisor y la valoración de las prácticas respecto a la formación y al desarrollo profesional de todos los agentes implicados en ellas : los alumnos, los profesores tutores y los profesores supervisores. La segunda, hacía referencia a la valoración del seminario de investigación-acción como escenario para la formación profesional. Concretamente, nos interesaba saber la valoración que hacían del trabajo colaborativo los profesores tutores, supervisores y colegas respecto a la elaboración de los materiales curriculares, al análisis de los vídeos de las sesiones previamente planificadas en el seminario de investigación-acción, y las ventajas e inconvenientes de la investigación-acción colaborativa en la formación inicial y permanente del profesor tutor y supervisor.

A los alumnos, futuros profesores, les planteamos además otras cuestiones para ver cuáles eran sus percepciones más significativas, sus creencias y sus principales dilemas respecto a las prácticas de enseñanza. Indagábamos, después de realizar las 
prácticas, sobre su formación, sus carencias, la relación entre el currículum formativo de la Escuela Universitaria y los proyectos educativos de las escuelas, el papel de las didácticas específicas, concretamente la Didáctica de la Educación Física y la importancia de la colaboración con otros colegas.

Todas las entrevistas fueron grabadas en audio y transcritas para posteriormente analizar la información aportada. Además de la entrevista, utilizamos para la recogida de información las grabaciones en vídeo de las sesiones realizadas, una cada semana, durante el mes que duraron las prácticas. Asimismo, también fueron grabadas en audio todas las reuniones del seminario que, posteriormente, han sido transcritas, con el fin de contar con una reproducción verídica de lo acontecido en el seminario.

En las reuniones del seminario, nos juntábamos todo el equipo durante dos horas una vez a la semana para analizar el vídeo correspondiente y valorar el proceso del propio seminario. Los temas más recurrentes eran aquellos que guardaban más relación con la práctica, desde la perspectiva técnica.

\subsection{Análisis e interpretación de la información}

El análisis e interpretación de los datos lo realizamos siguiendo los pasos de la espiral de la investigación-acción, de tal forma que iniciamos el proceso del análisis e interpretación con la propia investigación. Este proceso fue llevado a cabo siguiendo las pautas de las técnicas conceptuales de los análisis e interpretación de los datos, de Goetz \& Lecompte (1988,p.175). En nuestro estudio utilizamos más, concretamente, la teorización definida por estos autores como una forma genérica del pensamiento sobre el cual se construye el análisis, compuesta a su vez por la percepción, comparación, contrastación, agrupación y ordenación, establecimiento de vínculos y relaciones, especulación y los procedimientos analíticos generales, específicamente las comparaciones constantes, diseñadas por Glaser \& Straus (1967).

Basándonos en estas orientaciones, en un primer momento, comenzamos la reducción de la información analizando el contenido de la entrevista de cada uno de los profesores tutores y de cada uno de los alumnos, futuros profesores. En un segundo momento, analizamos, de forma conjunta, en relación con cada uno de los ítems que configuran cada uno de los dos bloques, la entrevista de los alumnos por un lado, y, por el otro, la de los profesores tutores. Seguidamente, procedimos al análisis de todos los datos aportados por los debates mantenidos en las distintas reuniones del seminario.

La entrevista de los alumnos tenía, entre otros, los siguientes objetivos: conocer cuáles eran sus pensamientos sobre la importancia de las prácticas como una oportunidad privilegiada para aprender a enseñar, saber que opinaban de su de formación y de sus carencias, recoger la mayor información posible acerca de la relación entre el currículum formativo de la Escuela Universitaria y los proyectos educativos de las escuelas donde realizaban las prácticas de enseñanza, averiguar sí la participación y la colaboración en el seminario de investigación-acción fue significativa para su formación docente. La entrevista a los profesores tutores tenía como finalidades: saber sus opiniones sobre la idoneidad en las relaciones que existen entre la institución universitaria y las escuelas antes, durante y después de las prácticas de enseñanza y conocer sus opiniones con respecto al seminario de investigación-acción como una herramienta de formación. 
La interpretación de la información derivada de las entrevistas a los alumnos y a los profesores tutores, y la procedente de las reuniones del seminario queda englobada en dos amplias categorías que desarrollaremos, en dos puntos, en el apartado siguiente de las conclusiones.

\section{CONCLUSIONES}

Las principales conclusiones a las que nosotros hemos llegado, después de haber hecho la codificación y la triangulación de la información recopilada en las entrevistas y en las reuniones de seminario, están sintetizadas en los dos puntos siguientes.

\subsection{La relación Universidad - Centros Educativos y supervisor-tutor}

Los alumnos, futuros maestros especialistas de Educación Física, consideran insuficiente la relación entre el currículum formativo inicial de la escuela universitaria y el Proyecto Educativo de los respectivos centros escolares donde realizan las prácticas. Tienen la sensación de que en su formación hay como dos itinerarios distintos, uno, que sitúan, en primer lugar, en la Escuela Universitaria y otro que corresponde a las prácticas de enseñanza en los centros escolares. De tal forma que la famosa relación teoría y práctica sigue estando encerrada, metafóricamente hablando, en el baúl de las ideas, por varios candados. Después de algunos años empeñada en saber quién tiene las llaves, continúo sin tener información suficiente para descubrir este gran dilema. Sin embargo, he llegado a la conclusión, retomando la metáfora, que una de las llaves está en manos de la Universidad y de la Administración Educativa. Es a estas entidades a las que les corresponde dotar de medios y crear las condiciones necesarias para que los profesores supervisores, tutores y alumnos puedan reflexionar colaborativamente y superar así la laguna que separa esos dos mundos: el de la teoría y el de la práctica. En este sentido, resulta muy esclarecedor un comentario expuesto por una de las maestras y compartido por todo el equipo:

A nosotros, los maestros, nadie nos paga el trabajo que conlleva estar ahora en este seminario, estamos dedicándole un tiempo fuera de la jornada escolar y cuando llegamos aquí ya estamos rendidos.

Nos parece importante resaltar que algunas opiniones hacían alusión a la falta de incentivos para que los profesores adopten un papel más reflexivo sobre su propia práctica, colaboren con colegas e intercambien experiencias, incluso, con otros centros. A modo ilustrativo, mencionaremos un comentario expuesto por una de las maestras, en el sentido de cierta crítica, respecto a las prácticas de años anteriores.

Había algunos tutores que querían que se negociara el aceptar alumnos en prácticas a cambio de ciertas cosas. La universidad tenía que ofrecer algo a cambio, había lo de las matrículas gratuitas y después de negociar conseguimos que nos reconocieran treinta horas para los sexenios. Para eso me voy a un cursillo y no tengo un alumno de prácticas que me esté controlando. Muchos maestros creen que los supervisores envían a los alumnos para controlar, así que yo los entiendo. 
En la universidad, la actividad de los supervisores no está suficientemente reconocida ni valorada. En consecuencia, la función de los supervisores de ayudar a los futuros profesores a relacionar la teoría y la práctica queda, en muchas ocasiones, reducida a la evaluación "burocrática" de la memoria de prácticas.

Continuando con la metáfora de las llaves, una segunda pensamos que está en la relación entre el supervisor y el tutor, que en muchos casos puede calificarse de burocrática. Para que la llave habra la puerta de la relación dialéctica entre la teoría y la práctica se precisa de una mayor colaboración de los profesores implicados: supervisor y tutor. El período de prácticas debería estar planificado por las instituciones y por los profesores implicados en ellas. Desde esta perspectiva deben entenderse como una situación privilegiada para la formación, no sólo del futuro profesor, sino también del profesor tutor y supervisor.

No son pocas las voces que proclaman la idea de la colaboración entre los centros, el profesorado y las universidades como un avance para la investigación del desarrollo de la formación continua e inicial del profesorado. Esta reflexión exigiría ser complementada con la referente al status que tienen los profesores tutores y supervisores implicados. Hemos podido constatar, en nuestra investigación, que los profesores supervisores Ilevan la iniciativa, no sólo a la hora de presentar el proyecto sino también durante todo el proceso. Los tutores adoptan una postura más receptiva y sienten un gran interés por todos los temas relacionados con la práctica docente. Cabe mencionar un comentario muy revelador realizado por una de las tutoras a una de nuestras primeras intervenciones, en las que exponiamos el marco teórico del proyecto y una ejemplificación didáctica sobre el tema de la expresión corporal.

Este cachito, en referencia a la ejemplificación didáctica, es lo que a mi me ha motivado, sólo por este ratito ya vale la pena estar aquí.

Como se ha podido comprobar en este comentario anterior, los tutores resaltan las propuestas curriculares prácticas como fuente de motivación para participar en el proyecto.

Una tercera llave, no por orden de importancia, está en manos de los formadores de profesores. A pesar de que la preparación de profesores reflexivos ha sido una de las principales metas en los últimos años de los programas de formación todavía queda mucho camino por recorrer para alcanzar el objetivo pretendido. Existe un asenso bastante generalizado de que el futuro profesor debe desarrollar su capacidad reflexiva, aprender por sí mismo, partiendo de la comprensión, de la exploración activa, del descubrimiento personal y de la colaboración con otros colegas. Sin embargo, en algunos casos no se crean las condiciones ni los contextos adecuados para la reflexión y la comunicación, de tal forma que los alumnos tienen pocas oportunidades para desarrollar y refinar la reflexión colaborativamente. En este sentido, uno de los alumnos del equipo arguye:

Yo entiendo lo que tu dices, con relación a la reflexión, pero la realidad en el día a día es totalmente diferente, por ejemplo a mí ni en la escuela, ni en el instituto, ni ahora en la universidad me han enseñado a cuestionar nada de 
nada, seguimos tomando apuntes para estudiar memorizándolos lo mejor posible para luego saber contestar en el examen[...]

Desde los programas de formación hay que potenciar y animar al alumno a aprender por sí mismo, partiendo de la comprensión, de la exploración activa y del descubrimiento personal. El comentario anterior nos da pie para comentar que los formadores de profesores deberían a través de la metodología que empleen en sus clases facilitar más la colaboración, el análisis y la reflexión.

Potenciar y reivindicar la reflexión en la acción y sobre la acción de cara a formar un profesor reflexivo y crítico que analice su práctica diaria, que seleccione diferentes alternativas de acción acordes con las situaciones reales, complejas, cambiantes e irrepetibles. Un profesor comprometido que comparta sus experiencias con el colectivo docente, de modo que su actuación reflexiva le guíe hacia la construcción y reconstrucción del conocimiento, modificando las ideas, creencias, teorías profesionales y valores sobre la enseñanza, han sido las metas de nuestro proyecto. Hemos puesto un gran empeño en apoyar y crear el clima para, junto a los alumnos, futuros profesores, y las maestras, reflexionar colaborativamente sobre el proceso de enseñanza-aprendizaje y sobre problemas relativos al contexto educativo y a la educación como configuraciones sociales inherentemente políticas e ideológicas.

\subsection{La valoración del seminario de investigación-acción}

Teniendo siempre presente la idea de conseguir formar un profesional reflexivo, consideramos que nuestro seminario de investigació-acción, donde colaboramos los supervisores, los tutores y los alumnos en prácticas, ha sido un buen escenario para vincular la teoría y la práctica, y estimular la reflexión en unión estrecha con la práctica docente y la innovación educativa. Si embargo, es preciso señalar que los obstáculos o dificultades que hemos encontrado, hace ya una década, en otro seminario de similares características, son prácticamente los mismos con los que nos hemos topado en esta experiencia (véase García Ruso, 1993). Esta situación real nos induce a pensar que la cultura que subyace en las instituciones implicadas no es fácil de cambiar.

Con la implantación de la Ley de Ordenación General del Sistema Educativo (LOGSE) y con la reforma de los planes de estudio de magisterio se pretendía de manera explícita formar un profesor reflexivo, crítico, con autonomía profesional. De un profesional de la educación adornado con esos calificativos sería de esperar que mudase las ideas, no siempre válidas, recibidas como alumno, que cambiara la cultura de la individualidad por la de la colaboración, que relacionase dialécticamente la teoría y la práctica, que se involucrase en los cambios por una sociedad más justa, que justipreciase además de los contenidos conceptuales, los procedimentales, las actitudes y los valores, que elaborase proyectos curriculares que se adapten a las características y necesidades de los alumnos, escuela y sociedad. No obstante, a pesar de estas buenas intenciones, la realidad es bien distinta, las causas del desajuste entre lo pretendido, formar un profesional reflexivo con autonomía, y lo alcanzado, hay que buscarlas en varios frentes. En esta última década el tema que aquí nos ocupa ha sido debatido en muchos congresos, en publicaciones y tesis doctorales de distintas disciplinas, pero cuando volvemos después de unos años a reto- 
mar el tema de las prácticas docentes percibimos que nada o muy poco se ha avanzado en la dirección pretendida. El tener, o no, autonomía no depende sólo de la voluntad y conocimientos del docente. Las condiciones reales en que se desenvuelve su tarea, así como la cultura escolar en la que se circunscribe, son factores que la favorecen o dificultan. Las palabras que siguen de Escudero (1999) apoyan muy bien el comentario anterior:

Incurrimos en un riesgo si, a estas alturas, no logramos advertir debidamente la complejidad que afecta incluso a las mejores ideas de transformación, cuando se depositan sobre ciertos contextos institucionales y profesionales sin percatarse de las tradiciones, culturas y regularidades que no sólo ordenan sus mundos de sentido sino también unos y otros mecanismos y poderes encargados de perpetuarlos (p.135).

Las estructuras institucionales que modelan, en cierta medida, las prácticas de los profesores y de los formadores de los profesores en sus centros respectivos, modelan también sus pensamientos y las tomas de decisiones sobre dichas prácticas. La cultura balcanizada donde prima el dominio de la especialización de materias que se caracterizan por los límites firmes y persistentes que se establecen entre los distintos estamentos de la organización académica han sido y siguen siendo un obstáculo que hay que salvar para llegar, una vez superadas otras trabas personales, institucionales y contextuales, a una cultura de la colaboración. Analizar y comprender los límites de la propia práctica profesioanal es una condición esencial para entender muchas de las limitaciones y posibilidades del desarrollo profesional y del cambio educativo.

Una de las limitaciones que ha estado presente desde el primer día ha sido la relacionada con el tiempo para la colaboración y la reflexión. Este hecho queda recogido de forma muy sucinta en los siguientes comentarios.

Deberíamos tener dos tardes libres para poder reflexionar y hacer este tipo de trabajo. [...] A mi el Director me dejaría salir antes pero va haber en el claustro quien proteste

Es una constatación común entre los implicados que el seminario de investigación-acción es una situación idónea para su desarrollo profesional. En las sucesivas reuniones del seminario los profesores supervisores, tutores y los alumnos, futuros profesores, van descubriendo nuevas maneras de enfocar la práctica profesional y, en tal sentido, cosideran muy positiva la planificación de la unidad didáctica de expresión corporal, la puesta en práctica de la misma, la grabación de las sesiones (entendiendo por sesión una secuencia de actividades organizadas coherentemente en función del nivel del grupo clase, del tiempo disponible y de unas intenciones previamente definidas) y el posterior análisis de los vídeos de las sesiones grabadas.

Los aspectos más sobresalientes en relación con la dinámica del propio seminario coinciden, prácticamente, con otros observados en la experiencia desarrollada en años anteriores por García Ruso (1993). Uno de los hándicaps a resolver es el recelo o la falta de confianza que experimentan los tutores y los futuros profesores a la hora de dialogar y comunicar a todos los miembros del seminario los dilemas a los 
que se enfrentan, día a día, en su labor docente. Los dilemas abarcaban un amplio abanico que van desde los problemas técnicos: disciplina, gestión de la clase, tratamiento de la diversidad, métodos de enseñanza, evaluación... hasta problemas administrativos, pasando por las relaciones laborales con otros colegas.

Los problemas técnicos a los que hemos hecho alusión anteriormente son los que están más presentes a lo largo de todas las reuniones del seminario de in vestigaciónacción. La pretensión más notoria era la consideración del seminario como un lugar donde pretendían se les ayudase a enseñar. Pese a nuestra constante insistencia a lo largo de los debates, de un análisis y reflexión de la propia acción práctica de los profesores, tal reflexión no alcanzo las cotas deseadas por nosotros, dado que en la mayoría de los casos sus reflexiones se ceñían a la aplicación técnica de contenidos procedimentales. Tanto el profesor tutor como el alumno, futuro profesor, se interesaban más por aquellos aspectos relacionados con las actividades, la adecuación de las tareas, la gestión, el ritmo y continuidad de la clase. Hay que destacar algunas reflexiones muy fructíferas que se centraban en la integración de dos alumnos minusválidos y en la propia estrategia metodológica empleada por las tutoras. Nuestra propia experiencia en el ámbito de la investigación-acción nos ha posibilitado la adquisición de ciertas estrategias para reconducir los problemas técnicos, con un sesgo claramente utilitarista, hacia otros más críticos. Sin embargo, el intento por nuestra parte de fomentar un debate más reflexivo y, a la vez, crítico, no siempre obtuvo respuestas positivas por parte de todos los miembros implicados, sino que éstos consideraban con cierta suspicacia los comentarios que hacían el proceso de enseñanza aprendizaje más complejo, al suscitar temas macrocontextuales con un marcado carácter colectivo y no meramente individual.

La valoración que sobre el seminario de investigación-acción realizaron todos los miembros implicados fue muy positiva. Las tutoras consideraron muy importante el proceso seguido, que les brindó muchas oportunidades para compartir experiencias docentes innovadoras y problemas relacionados con la organización escolar y el estatus laboral.

Los alumnos, futuros profesores, compartieron con las tutoras y con las supervisoras la responsabilidad en la toma de decisiones acerca de la planificación e implementación de las actividades docentes. Los objetivos de las prácticas referentes a desarrollar en el alumno, futuro profesor, la capacidad de análisis del trabajo realizado en la escuela, la identificación de los aspectos implicados en el proceso de enseñanza-aprendizaje, la toma de decisiones preactivas e interactivas, el análisis de su propia actuación y de los demás implicados en el proyecto, han sido alcanzados. Su rol no sólo era observar y recibir pasivamente conociminetos prácticos sino que participaban activamente en todo el proceso. Las tutoras animaban a los alumnos en prácticas para que fueran ellos los que impartieran las sesiones de expresión corporal, colaborativamente planificadas, el papel de observador recaía en las tutoras, quienes tomaban notas, en un primer momento, en una ficha de observación que tenía como objetivo focalizar la atención en aspectos relevantes de la práctica docente (presentación de la sesión, descripción de la actividad, dificultad e intensidad de la tarea, continuidad y ritmo de la clase, calidad de la participación de los alumnos, estilo de enseñanza, interacción, organización, motivación, feedback...) y posteriormente de manera más abierta. Al finalizar la sesión la tutora y el alumno comentaban la experiencia, el proceso seguido y después en la reunión del semina- 
rio compartían con los demás miembros del seminario sus vivencias relativas a la puesta en práctica de la sesión.

Los miembros del equipo son partidarios de continuar colaborando, en años próximos, en las prácticas de enseñanza. Consideran la experiencia del proyecto de investigación-acción muy interesante, aperturista, innovadora, con un gran potencial para la investigación educativa y para el desarrollo profesional de los profesores. Como colofón final, señalar el comentario de una maestra tutora:

La investigación-acción nos muestra nuevos caminos para la enseñanza, nuevos significados, renueva nuestra manera de pensar y ver la enseñanza. Hace que no caigamos en la rutina profesional, donde se da todo por aprendido. El intercambio de experiencias que se favoreció entre nosotros me sirvio para desarrollar nuevos conocimientos y experimentar aquellas propuestas elaboradas por todo el grupo con muchas ideas que a mi sola nunca se me ocurririan.

\section{BIBLIOGRAFÍA}

ALMOND, L. (1997) Reflecting on practice: A changing research rale. Quest, 49, 394-402.

BLÁNDEZ, J. (1996) La investigación- acción, un reto para el profesorado. Guía practica para grupos de trabajo, seminarios y equipos de investigación. Barcelona: Inde.

CARR, W. y KEMMIS, S. (1988) Teoría crítica de la enseñanza. La investigaciónacción en la formación del profesorado. Barcelona: Martínez Roca.

CARREIRO, F. (1994) La formación del profesorado de Educación Fïsica: ¿qué objetivo?, ¿qué contenidos?, ¿qué método?. Actas XI Congreso nacional de Educación Fïsica en Escuelas Universitarias de magisterio, 1-14. Segovia: Universidad Autónoma de Madrid.

DÍAZ, B. y MARTÍNEZ, L. (1994) Las prácticas específicas de enseñanza dentro de la formación inicial de los especialistas de Educación Física. En Actas XI Congreso Nacional de Educación Física de Escuelas de Magisterio, 96-101. Segovia: Universidad Autónoma de Madrid.

ELLIOTT, J. (1990) La investigación-acción en educación. Madrid: Morata.

ELLIOTT, J. (1993) El cambio educativo desde la investigación-acción. Madrid: Morata.

ESCUDERO, J. M. (1999) La formación permanente del profesorado universitario: cultura, política y procesos. Revista Interuniversitaria de Formación del Profesorado, 34, 133-157.

FRAILE, A. (1997) El prácticum como actividad de formación colaborativa. En D. Ayora, et al. (Eds.) Aportaciones al estudio de la actividad física y el deporte. Valencia: INEV

GARCÍA RUSO, H.M. (1993) La formación del profesorado de Educación Física: una propuesta de curriculum basada en la reflexión en la acción. Santiago: Servicio de Publicaciones, Universidad. 
GARCÍA RUSO, H.M. (1997) La formación del profesorado de Educación Física: problemas y expectativas. Barcelona: Inde.

GARCÍA RUSO, H.M. (2002) El prácticum herramienta para la investigación y la formación en la Educación Física. Santiago de Compostela: Servicio de Publicaciones, Universidad.

GLASER, B. y STRAUSS, A. (1968) The discovery of grounded: strategies for qualitative research. Chicago: Aldine.

GOETZ, J.P. y LECOMPTE, M.D. (1988) Etnografía y diseño cualitativo en investigación educativa. Madrid: Morata.

GRAHAM, K.C. (1991) The influence of Teacher Education on preservice development: Beyond a custodial orientation. Quest, 43 (1), 1-20.

HARDY, C.A. (1999) Preservice Teachers perceptions of learning to teach in a predominantly school - based. Teacher Education Program. Journal of Teaching in Physical Education, 18, 175-198.

KEMMIS, S y MCTAGGART, R. (1988) Cómo planificar la investigación-acción. Barcelona: Laertes.

MARCELO, C. (1995) Investigaciones sobre prácticas en los últimos años: Qué nos aportan para la mejora cualitativa de las prácticas. En MONTERO, L. y otros (eds.) El prácticum en la formación de profesionales: problemas y desafíos, 338-361. Santiago: Torculo.

MARCELO, C. y ESTEBARANZ, A. (1998) Modelos de colaboración entre la Universidad y las Escuelas de formación del profesorado. Revista de Educación, 317, 97-120.

MARTÍNEZ, L. (2001) Los seminarios de seguimiento del prácticum de Educación Física; una ayuda para fomentar y orientar la reflexión. En Resumenes de VI Simposium Internacional sobre el Prácticum. Desarrollo de competencias personales y profesionales en el prácticum,.180-183. Lugo: Unicopia.

MARTÍNEZ, L. y VACA, M. (1999) El tutor universitario como enlace entre el plan de prácticas y su desarrollo en los centros. En SÁEZ, P. y otros (Coords) XVII Congreso Nacional de Educación Física,113-128. Málaga: Instituto Andaluz del Deporte.

MAXWELL,J.A. (1998) Designing a Qualitative Study. En BICKMAN, L. y ROG, D. J. (Eds.) Handbook of applied social research methods, 69-100. London: Sage.

MCKERNAN, J. (1999) Investigación-acción y currículo, Madrid: Morata.

MONTERO, M.L. (1990) Modelos de prácticas: modelo implícito de prácticas que revelan los actuales programas de formación inicial del profesorado. En ZABALZA, M.A. (Coord). La formación práctica de los profesores, 41-52. Santiago: Torculo.

ORTIZ, M.M. y ROMERO, C. (2000). El prácticum en la especialidad de Educación Física. Planteamientos y reflexiones de los diferentes agentes implicados. En CONTRERAS, O. La Formación inicial y permanente del profesor de Educación Física, 181-191. Ciudad Real: Servicio de Publicaciones de la Universidad de Castilla la Mancha. 
O'SULLIVAN, M. y TSANGARIDOU, N. (1997) The role of reflection in shaping physical education teacher,s educational values and practices. Journal of Teaching in Physical Education, 17, 2-25.

PAIVA, M.L. (1998) Autoformacâo e transformacâo das prácticas professionais dos profesores. Revista de Educaçao, vol. VII, nº 2, pp 53-61.

ROMERO, C. (1995) Orientaciones sobre el Plan de Prácticas de enseñanza del maestro de la especialidad de Educación Física. Granada: Promeco

ROVEGNO, I. (1992) Learning to reflect on teaching: A case study of one preservice physical education teacher. The Elementary School Journal, 92, 491-510.

STENHOUSE, L. (1975) An introduction to currículum research and development. London: Heinemann.

STENHOUSE, L. (1987) La investigación como base de la enseñanza.Madrid: Morata.

TINNING, R. (1992) Reading action research: Notes on knowledge and human interest. Quest, 44, 1-14.

TINNING, R. (1994) Enseñanza reflexiva, investigación - acción y formación del profesorado de Educación Física. En Actas XI Congreso Nacional de Educación Física en Escuelas Universitarias de Magisterio, 15-19. Segovia: Universidad Autónoma de Madrid.

TOJA, M.B. (2001) Estudio de un programa de formación en preservicio en Educación Física: La investigación-acción como estrategia de formación e investigación. (Tesis doctoral).

TSANGARIDOU, N. y O'SULLIVAN, M. (1994) Using pedagogical reflective strategies to enhance reflection among preservice Physical Education teachers. Journal of Teaching in Physical Education, 14, 13-33.

VICIANA, J. (1996) Evolución del conocimiento práctico de los profesores de Educación Física en un programa de formación permanente colaborativo. Universidad de Granada (Tesis doctoral).

ZABALZA, M.A. (1993) La formación en alternancia como respuesta a la relación teoría - práctica: Dilemas curriculares surgidos en la experiencia de los últimos años. En MONTERO, L. y VEZ, J.M. (Eds). Las didácticas específicas en la formación del profesorado, 131-149. Santiago: Torculo.

ZEICHNER, K.M y NOFFKE, S.E. (2001) Practitioner Research. En Richardson, V. (Ed.) Handbook of Research on Teaching, (4 ${ }^{\text {th }}$ ed.), 298-330. Washington. American Educational Research Association. 